Revue européenne des sciences sociales

European Journal of Social Sciences

La sociologie en quête d'une théorie générale

\title{
Entités sociales collectives, représentations collectives et réseaux
}

Pierre Livet

\section{(2) OpenEdition}

Journals

Édition électronique

URL : http://journals.openedition.org/ress/174

DOI : $10.4000 /$ ress. 174

ISSN : 1663-4446

Éditeur

Librairie Droz

Édition imprimée

Date de publication : 1 février 2008

Pagination : 133-151

ISBN : $978-2-600-01221-8$

ISSN : 0048-8046

Référence électronique

Pierre Livet, "Entités sociales collectives, représentations collectives et réseaux 》, Revue européenne des sciences sociales [En ligne], XLVI-140 | 2008, mis en ligne le 01 février 2011, consulté le 01 mai 2019. URL : http://journals.openedition.org/ress/174 ; DOI : 10.4000/ress.174 


\section{Pierre LIVET}

\section{ENTITÉS SOCIALES COLLECTIVES, REPRÉSENTATIONS COLLECTIVES ET RÉSEAUX}

\section{INTRODUCTION}

Il est tentant de décrire les relations sociales dans les termes de réseaux d'interactions. En effet ces interactions devant être effectives, on peut penser avoir alors affaire à une description de la société dans son actualité. On échappe ainsi au constructionnisme radical qui voit dans la société le seul résultat des représentations des individus. Mais le problème est que les individus entretiennent effectivement des représentations du collectif, qui sont souvent elles-mêmes collectives - au moins au sens de partagées par l'ensemble des individus- et que ces représentations collectives influencent les interactions.

Nous nous proposons dans ce travail de partir des réseaux d'interactions. Pour traiter le problème des représentations, nous ne nous satisferons pas de rajouter des représentations aux interactions. Nous tenterons de définir les conditions auxquelles ces interactions en réseau produisent des entités collectives, qui sont alors le fondement et le référent des représentations collectives. L'épistémique des représentations pourra alors s'appuyer sur l'ontologique d'entités collectives. Nous les définirons comme des sommets virtuels du réseau, et il faudra donc déterminer à quelles conditions un réseau peut produire un sommet virtuel. Quand les individus utiliseront ces représentations collectives, leur influence tiendra à ce qu'elles seront justifiées par la structure même des interactions du réseau. Il sera même alors possible de définir les conditions auxquelles une représentation $d u$ collectif - et non plus simplement une représentation collective- est justifiée et donc a une efficience dans le réseau social.

Dans un premier temps le formalisme des réseaux peut apparaître décevant pour ce qui concerne notre problème des entités collectives et de leurs représentations. Notre déception est en rapport avec les espérances qu'ils suscitent. Ainsi, les transformations possibles sur leurs graphes peuvent révéler des classes (au sens mathématique), et diverses propriétés structurelles (équivalence structurale, équivalences régulières, etc.). Mais le problème est alors celui du rapport entre ces propriétés formelles et une interprétation dans des termes sociaux, comme identité de groupe, ou relation de pouvoir. On ne peut pas prétendre par exemple que pour chaque trou structural ${ }^{1}$ il y a relation de pouvoir (la notion structurelle n'est pas

1 Un sommet d'un graphe devient un lieu de décision de transmission parce qu'il manque des liens (trou structural) entre les autres sommets auxquels il est relié, liens qui permettraient de faire passer les interactions et informations sans utiliser le sommet central. 
suffisante), ni que pour toute relation de pouvoir il y a trou structural, ou bien il faudrait montrer que cette notion structurelle est nécessaire à la notion de pouvoir, ce qui serait délicat, et même impossible. Cela tient à ce que les propriétés structurelles des réseaux ne sont pas forcément réinvesties dans les relations sociales actuelles, de manière à y être efficientes socialement. Elles peuvent rester formelles. Au contraire, les notions de groupe, de communauté, d'institution, etc. s'appuient bien sur des propriétés structurelles -qui elles-mêmes reposent sur des interactions actuelles-, mais elles ne restent pas formelles: on peut supposer qu'elles ont une efficace sur les interactions actuelles. Du coup, elles ne peuvent plus se réduire à des propriétés formelles tirées des interactions actuelles de base.

Il est alors difficile de garantir la continuité de sens entre l'interprétation d'une interaction prise pour une donnée et l'interprétation des propriétés structurelles que les transformations nous révèlent. Or cette continuité est cruciale pour notre problème des conditions d'émergence des «nous ». Cette difficulté, on la trouve par exemple chez Forsé. Pour nous introduire à l'analyse des cercles sociaux, il nous explique comment on peut construire des matrices qui ne se bornent pas à indiquer dans chaque case la présence ou l'absence d'interaction entre deux individus, mais qui peuvent indiquer le couplage entre un individu et un attribut, voire, via l'individu, entre deux attributs. Selon les répartitions des 1 et de 0 dans ces matrices, on peut distinguer des relations qui forment des cliques, ou encore des relations entre deux groupes différents, ou bien des relations qui vont de tout individu d'un groupe avec au moins un autre de l'autre groupe (blocs réguliers), ce qui peut correspondre au fait que les individus du premier groupe jouent un certain rôle pour les individus du second. Mais ensuite Forsé nous dit (à juste titre) que le concept de cercle social, qu'il veut tirer de ces relations matricielles, doit être fondé sur l'interreconnaissance des individus entre eux ${ }^{2}$. Or l'interreconnaissance est un concept qui n'a pas été entré en donnée, et dont on ne voit pas comment les découvertes structurelles que nous permettent les matrices pourraient nous le fournir en sortie. Il faut pour cela réinterpréter ces structures matricielles et admettre que l'interreconnaissance peut venir par exemple d'une combinaison de cohésion (cliques) et d'identité (possession d'un même attribut). Mais elle peut venir aussi bien d'une complémentarité de rôles (Forsé ne donne d'ailleurs pas de principe de clôture de la liste de ces conditions), et on ne voit pas en quoi ce complexe d'interprétations a été validé par la structure matricielle. Le problème est ici que les interactions sont des observables (même si cela passe par des réponses à des questionnaires, mais ces réponses sont elles-mêmes des interactions observables), que l'interreconnaissance est un partage de représentations, et que nous ne savons pas comment garantir que les représentations exprimées sont bien les représentations efficientes, celles qui guident les activités observables (en vertu du problème de la double intentionnalité: ainsi on peut accomplir une action pour tromper un adversaire, avec une intention seconde).

2 Alain Degenne et Michel Forsé, Les réseaux sociaux, Armand Colin, 2004, p. 217. Nous pourrions aussi faire référence aux travaux d'Emmanuel Lazega (Réseaux sociaux et structures relationnelles, PUF Que Sais-Je, 1998) et de Jean-Benoît Zimmerman, par exemple Zimmerman, J.B. «Cumul d'influence et réseaux sociaux: une application aux processus de diffusion de l'innovation», Revue d'Economie Industrielle, $N^{\circ}$ 96, 2001, pp.7-27 (avec F. Deroïan). 
On pourrait vouloir résoudre ces difficultés en faisant des notions de collectivités sociales de simples idées, ou encore le produit de représentations individuelles partagées. Ce serait leur interdire d'exercer des contraintes sur les interactions actuelles, sauf par le biais de la compréhension individuelle de ces représentations. Mais alors ce serait l'influence de chaque représentation individuelle qui aurait de l'effet, et ce ne serait pas l'existence des collectifs sociaux qui permettrait le développement d'interactions entre individus impossibles sans elles. Or il ne semble pas suffisant de considérer une institution ou une société comme une simple construction mentale qui dépende seulement de ce que des individus partagent un même ensemble de représentations. Supposer le collectif déjà réalisé par un «savoir mutuel» ou «common knowledge » revient à se donner le problème résolu ${ }^{3}$.

Inversement se donner le tout de la société, à la fois comme environnement culturel qui définit les significations disponibles pour l'acteur social et comme facteur déterminant ses interactions possibles, c'est se rendre impossible l'analyse de ces déterminations, puisqu'elles devraient aussi déterminer les activités de catégorisation du sociologue qui tenterait de les distinguer, si bien que soit il ne ferait que répéter un consensus social et son travail n'aurait alors pas d'intérêt, soit il exprimerait le point de vue d'une partie de la société, et son travail serait plus politique que scientifique. Il semble souhaitable d'échapper à ces combinaisons caricaturales de l'individualisme et du constructionnisme, d'un côté, et du holisme et de la détermination en dernière instance, de l'autre.

Pour ce faire, nous proposons de voir dans les entités collectives, dans les «nous », des entités virtuelles, et de faire une place dans notre conception du réel au virtuel comme à l'actuel ${ }^{4}$, au lieu de confondre le virtuel avec de l'idéel ou de la simple représentation. Le virtuel en ce sens ne se réduit pas à du formel. Le formel s'extrait de l'actuel sans influer sur lui en retour. Le virtuel «ordinaire», qui est simplement un «inactuel» provient de l'actuel (ce qui est passé est maintenant virtuel, mais a été actuel, et il en est de même de ce qui sera), et il n'influe donc sur l'actuel proprement dit que dans la mesure où il a été ou sera actuel. Cependant le virtuel que nous visons ne doit pas pouvoir se réduire à de l'actuel, contrairement à l'inactuel, et il doit pouvoir avoir une influence sur l'actuel, contrairement au formel ${ }^{5}$. C'est ce statut ontologique que nous recherchons pour nos entités sociales. Nous retrouvons ici un vieux problème: comment définir un statut du collectif qui reconnaisse sa différence par rapport aux individus qui le composent ${ }^{6}$ sans pour autant créer une entité imaginaire ou formelle et qui soit sans effet sur ces individus; le danger opposé serait de se donner le collectif dans son irréductibilité, ce qui rend inversement difficile de comprendre comment il peut influer sur les individus, tant il leur est hétérogène. La notion de virtuel est une tentative pour recon-

3 La notion de common knowledge est en revanche très utile pour déterminer ce que peuvent être les incomplétudes et défauts d'une situation de communication par rapport à cet idéal du savoir mutuel.

4 En suivant en cela Granger.

5 Ce virtuel est donc en situation d'émergence par rapport à l'actuel, puisqu'il provient de l'actuel mais qu'il ne s'y réduit pas et qu'il a le pouvoir de l'influencer, au moins par la structuration qu'il permet.

6 Ce que Tuomela, Searle, Gilbert appellent un nous, un sujet pluriel qui soit une entité sui generis. 
naître cette irréductibilité sans couper le lien avec l'actualité des activités individuelles. Le problème est d'arriver à définir les conditions de cette existence d'une manière qui soit plausible, sociologiquement et ontologiquement. Nous devrons pour cela recourir à l'idée de relation conditionnelle et donc de disposition. Mais nous nous appuierons sur des dispositions qui ont une efficace causale et qui sont donc reliées plus fermement à l'actualité que les simples représentations. Nous aurons aussi recours aux outils mêmes qui nous ont déçu dans un premier temps, les réseaux et leurs graphes, et cela pour pouvoir mieux déterminer les conditions auxquelles dans un graphe un sommet ou un lien virtuel peut se constituer.

Nous allons donc tenter de déterminer des conditions concernant les interactions fournies comme données pour le graphe d'un réseau qui soient telles qu'elles nous permettent de contourner ce problème, parce qu'elles nous permettront de présupposer une entité collective même en l'absence de représentations de cette entité. Il nous faudra bien entendu ensuite préciser le rôle que peuvent avoir les représentations du collectif, mais ce ne sera pas notre point de départ.

Dans un premier temps nous allons déterminer les conditions de constitution d'une entité virtuelle dans un réseau, et tester ces conditions à l'aune de nos intuitions sur les types de groupements qui méritent le nom d'entités sociales collectives. Dans un deuxième temps nous analyserons les relations entre les entités sociales collectives, les représentations sociales et les représentations des entités sociales collectives. Dans un troisième temps, pour mettre à l'épreuve les constructions et les catégories ainsi produites, nous réinterpréterons quelques positions sociologiques bien connues pour voir si nos catégories ont quelque fécondité interprétative. Le lecteur soucieux de raccorder nos concepts à des descriptions sociologiques peut donc passer directement de la première partie à la troisième, et revenir à la seconde pour considérer le rôle des représentations sociales.

\section{LES CONDITIONS \\ D'UNE ENTITÉ COLLECTIVE VIRTUELLE DANS UN RÉSEAU}

Partons d'un graphe très simple. Il nous permettra d'envisager diverses conditions pour l'existence d'un «nous », et de montrer à chaque fois en quoi elles sont insuffisantes, jusqu'à ce que leur ensemble nous paraisse satisfaisant. Supposons que nous ayons 5 éléments (des individus, mais ce pourrait être aussi des activités, un individu pouvant pratiquer différentes activités; nous pourrions par exemple avoir 3 individus et 5 activités). Supposons que ces activités forment une chaîne qui se boucle. Elles peuvent ainsi s'auto-entretenir les unes les autres collectivement. Nous avons là une base pour des interactions sociales. Nous ne nommerions pas «sociales», remarquons-le, des interactions dans lesquelles chaque individu peut directement contrôler ${ }^{7}$ et influencer tous les autres (ce qui n'est pas le cas

7 Nous donnons ici à «contrôler» un sens faible et pragmatique. Un premier individu contrôle l'activité d'un autre, eu égard à la réalisation d'une fin qui est utile au premier, si quand cette activité se détourne de cette fin ou ne lui est plus utile il peut par sa propre activité amener l'autre acteur à infléchir sa conduite dans le sens souhaité. Cette forme de contrôle exige de plus d'être directement en contact avec l'autre acteur. 
dans notre graphe). On ne voit d'ailleurs pas de situation sociale réelle qui satisfasse cette condition. Si l'on exigeait que le contrôle soit efficace, on ne pourrait pas aller bien plus loin que trois personnes, et même alors, les temps de repos nécessaires rendraient la continuité du contrôle irréalisable. Une «société » de ce type ressemblerait plutôt à un kidnapping mutuel.

Nos intuitions sur une société semblent donc exiger que certaines interactions soient indirectes, et que nous soyons dépendants d'activités accomplies par des personnes sur lesquelles nous n'avons pas de contrôle direct. C'est le cas dans notre chaîne qui se boucle: l'individu 1 contrôle directement ses voisins 2 et 5 , mais il ne contrôle pas 3 et 4 . Cependant, cette seule condition n'exige en rien la constitution d'une entité sociale virtuelle. Il suffit pour que le réseau perdure que la probabilité que les activités actuelles et effectives s'enchaînent en boucle soit suffisamment élevée. Il a sans doute pu ainsi exister des circuits de troc de tribu à tribu... etc. qui avaient une probabilité suffisante de se boucler mais qui n'avaient pas pour autant de statut social (si le circuit avait une dizaine d'éléments, la tribu 1 n'était pas influencée par le troc entre la tribu 4 et 5, par exemple). De même dans notre société, il peut se trouver qu'un circuit d'échange ait une probabilité suffisante, sans qu'il ait d'influence sociale autre que les bouclages effectifs qu'il assure. Dans ce genre de circuit, les individus qui le composent n'ont pas besoin de supposer une entité collective pour se représenter l'activité de ceux sur qui ils n'ont pas de contrôle direct ${ }^{8}$.

Mais ils n'en ont pas non plus besoin quand ils peuvent identifier tous les acteurs du circuit, y compris ceux sur qui ils n'ont pas de contrôle direct au moment où ces acteurs doivent accomplir l'activité requise pour le bouclage du circuit. Imaginons (nous sommes forcés ici d'imaginer: ce genre d'interaction n'existe pas dans une société réelle) une coordination dans laquelle il suffirait que chacun s'attende avec une fréquence suffisante à ce que les différents rôles assurés chacun par un individu identifié soient assurés. Le collectif du réseau se réduirait alors à l'ensemble des individus, plus les interactions et leurs fréquences. Chacun de ces éléments serait actuel à son tour, les fréquences seraient des sommations pondérées d'actualités. Aucun virtuel - aucune entité qui ne puisse pas se réduire à un ensemble d'éléments chacun actuel- ne serait nécessaire. La différence ontologique entre l'entité sociale et ses composants n'aurait pas lieu d'être.

Pour qu'un collectif soit nécessaire comme entité virtuelle, pour que nous nous référions à un «nous ", à un groupe, à un collectif, il faut ajouter à notre première condition, à savoir: 1) celle du bouclage de la chaîne, de l'existence d'un circuit dans le réseau, au moins deux autres conditions. 2) Il faut que le collectif tout entier soit nécessaire à chacun des individus - ou du moins à la majorité d'entre eux. Cette condition est déjà remplie par notre exemple de chaîne qui se boucle, puisque chacun des chaînons lui est indispensable. Dans la réalité sociale, cependant, aucune chaîne ne subsiste de manière isolée, les circuits sont toujours des parties d'un réseau plus complexe, et même ils sont ouverts sur des entrants externes au circuit. Il en est ainsi, si nous prenons un exemple dans des sociétés

8 C'est un cas où l'analyse en termes de réseaux pourra nous révéler l'existence du circuit, alors qu'il ne correspond pas à une entité collective, à un «nous». 
très réduites, du circuit de nourriture d'un petit groupe de chasseurs-cueilleurs, où la préparation et la conservation de la nourriture rapportée de la chasse ou de la cueillette sont nécessaires pour relancer les activités de chasse et de cueillette qui entretiendront aussi le circuit. Encore faut-il que le circuit soit alimenté de l'extérieur par les baies des arbustes (ou par des racines et bulbes) et par les proies de la chasse.

3) Il faut que pour chaque individu du réseau (pour ce qui concerne sa survie, ou son maintien dans le groupe, si nous le prenons comme acteur de base), il existe dans le circuit des activités dont l'accomplissement est nécessaire à son maintien dans la chaîne et qui sont telles qu'il ne puisse identifier quel individu les accomplit actuellement. Cela veut dire que même s'il peut identifier les individus susceptibles de les accomplir, ces individus sont substituables, et que la substitution effectuée lui échappe. Ainsi, pour notre acteur de base, l'individu dont dépend son maintien dans le groupe est un individu «marque-place». La survie de la femme qui après avoir fait la cueillette assure la conservation du gibier dépend du chasseur quel qu'il soit qui ramènera du gibier (même une fois tenu compte des différences matrimoniales), et de même celle du chasseur dépend du succès de la cueillette, qui que ce soit qui l'assure.

Cependant cette condition là non plus n'est pas suffisante: la place ainsi marquée reste définie par les autres activités de la chaîne comme un sommet qui a toujours une réalisation actuelle, puisqu'il est occupé par tel individu, ou par tel autre. Imaginons (là encore cela reste une fiction) un groupe de cueilleurs-chasseurs qui ne soit pas structuré, qui n'ait pas de rapport d'échanges (exogamiques, en particulier). Chacun d'eux pourrait compter sur les autres, sans se soucier de qui accomplit telle tâche, mais il lui suffirait de compter sur les individus membres du groupe, sans que le groupe en tant que tel ait un rôle dans cette coordination. Le groupe serait l'effet de la coordination, il ne jouerait pas de rôle causal.

Il faut donc exiger davantage. Il faut une quatrième condition: 4) l'occupation de cette place d'activité dans la chaîne doit être assurée par des membres reliés à ceux de la chaîne, dans un réseau, et de telle manière que si l'un d'eux était empêché de remplir cette activité, un autre le fasse. L'identité de celui qui assure cette activité n'est pas définie directement, elle est définie conditionnellement, par un système conditionnel d'indisponibilités des uns et de disponibilités des autres. Cette conditionnalité n'est plus réductible à une actualité, puisque quand la tâche est assurée par l'un, c'est conditionnellement au fait que l'autre ne l'assure pas. L'actualité est donc maintenant dépendante d'une non-actualité, dépendance qui ne peut pas être actuelle, et qui reste donc virtuelle. Mais il s'agit bien d'un virtuel actif, puisqu'il est la condition d'une actualité. Par exemple les chasseurs comptent sur les cueilleuses ou les cueilleurs en cas d'échec de leur chasse individuelle. Et les cueilleuses abandonnent temporairement leur tâche en cas de gibier abondant ramené par les chasseurs, pour en tirer le meilleur parti (utilisation des peaux, etc. $)^{9}$. Dans une famille nombreuse, les parents pris par d'autres tâches peuvent compter sur les aînés pour s'occuper des plus petits, et les aînés peuvent compter sur un des parents pour les relayer quand ils ont leurs propres travaux.

9 Les deux conditions ne sont pas réciproques, mais nous verrons que la réciprocité directe n'est pas nécessaire. 
Mais ce virtuel est-il bien collectif? Avons-nous déjà affaire à une entité du type d'un groupe? C'est semble-t-il le cas dans les deux exemples précédents. Est-ce le cas dans notre réseau à 5 éléments? Nos conditions sont satisfaites si une activité nécessaire pour que l'élément 1 continue à faire partie du circuit est par exemple assurée par l'élément 3 de manière conditionnelle au fait que l'élément 4 ne l'assure pas. Comme 3 et 4 sont en relations avec les mêmes sommets du graphe ( 2 et 5) ils sont structurellement équivalents. Cette équivalence structurale - jointe à la conditionnalité en question, et à l'impossibilité d'un contrôle directamène la constitution d'un sommet virtuel (on verra comment), qui est un marque place pour la substituabilité de 4 à 5 et qui est le lieu du lien conditionnel de l'un à l'autre. Pour être plus bref, nommons «dépendance au virtuel» ce quadruplet de conditions: impossibilité d'un contrôle direct, substituabilité, équivalence structurale et conditionnalité. Le sommet ou nœud virtuel n'existe dans notre graphe à 5 sommets que dans la relation entre 1 et $\{3,4\}$. L'élément 2 , lui, a affaire soit à 3 soit à 4 , il peut contrôler directement l'un ou l'autre, et il en est de même de 5 , si bien que 3 est 4 ne sont pas nécessairement pour eux virtuels.

Pour que le sommet virtuel ait l'existence qu'a un groupe pour ses membres, et non pas pour un seul de ses membres, il faut donc que soit satisfaite une cinquième condition: 5) Pour tout individu de la chaîne ou plus généralement du réseau, il faut qu'existe au moins un sommet virtuel créé par équivalence structurale et conditionnalité entre d'autres membres du réseau, relativement à cet individu (par souci de brièveté, nous parlerons de substituabilité ou d'équivalence conditionnelle). Un cas limite est celui où pour chaque acteur il existe une classe d'équivalence conditionnelle différente, et qu'elles ne sont pas reliées entre elles, si bien que l'entité collective pourra être définie différemment pour chaque acteur. Un cas limite opposé serait celui où chaque acteur est dépendant de la même classe d'équivalence conditionnelle, bien entendu à trois acteurs près (lui et les deux acteurs qu'il peut contrôler directement). La plupart du temps, ces dépendances au virtuel sont nécessaires au maintien dans la chaîne non pas d'un seul, mais de plusieurs éléments. Nos deux cas limites définissent donc un intervalle, et c'est la plupart du temps dans la partie médiane de cet intervalle que se trouveront nos prototypes de collectifs (les familles, les tribus, les clans, les équipes, etc.). On pourrait aussi imaginer une situation où chaque dépendance au virtuel relie deux éléments substituables conditionnellement et un acteur de base, où l'acteur de base et un des éléments sont à chaque fois différents, mais ces substituabilités conditionnelles toutes différentes sont cependant reliées parce que chaque individu de base joue aussi le rôle d'élément constituant d'une autre dépendance au virtuel. Nous aurions alors à la fois une participation de chacun au virtuel, et une entité virtuelle des plus distribuées.

Notons en quoi notre définition d'une entité collective virtuelle, d'un «nous », diffère de celle que l'on trouve par exemple chez un auteur comme Margaret Gilbert. Sa version est la suivante: deux individus (ou plus) se trouvent agir ensemble (son exemple est «marcher ensemble»), chacun s'attend à ce que cette coordination continue, et cette attente est de «common knowledge ${ }^{10}$. De là, elle

${ }^{10}$ Marcher ensemble, PUF, 2004 
infère que tant qu'aucun des partenaires n'a manifesté son intention de rompre la coordination, ils se trouvent chacun réellement engagés à la poursuivre.

Notre définition est à la fois plus et moins exigeante. Plus, parce qu'elle ne considère pas qu'une coordination temporaire entre deux personnes corresponde à nos intuitions de ce qu'est une entité collective. Il y faut plus de personnes, plus de réseau d'interactions, plus de substituabilité. Moins, parce que nous pouvons nous trouver membre d'une entité collective sans l'avoir voulu (on le verra dans le cas des jeunes des banlieues), sans que cela corresponde à nos attentes, et sans que nous puissions pour autant être assuré du «common knowledge » de la mutualité de ces attentes. Margaret Gilbert est obligée de compenser la faible dimension sociale d'une simple activité intersubjective temporaire par un renforcement d'engagement, renforcement qui dans son exemple du «marcher ensemble » n'est pas fondé et qui n'est pas non plus nécessaire pour imposer une collectivité.

Revenons à notre représentation par réseau. Pour représenter un nœud virtuel (nous désignons ainsi une convergence qui relie les sommets 3 et 4 au sommet de base 1) à partir du graphe de notre chaîne en boucle, nous avons en fait besoin de dédoubler le graphe de cette chaîne, et de relier les deux graphes par les conditionnalités correspondantes. Ainsi le sommet 2 va avoir un arc qui le relie au sommet 3 ; dès lors il ne sera pas relié actuellement au sommet 4 , ce qui nous symboliserons par un arc barré. Un arc barré reliera de même ce sommet 4 au sommet 5 (indiquant que l'interaction entre 4 et 5 n'a pas lieu, pas plus que celle entre 2 et 4 ). Ces deux arcs doivent être indiciés par référence à l'actualisation du sommet 3. De l'autre côté, le sommet 2 du graphe dédoublé va être relié au sommet 4 , et donc par un arc barré au sommet 3 , qui sera alors relié par un arc barré au sommet 5 du graphe dédoublé. Ces deux arcs sont indiciés par rapport à l'actualisation du sommet 4. En repliant les deux graphes l'un sur l'autre, nous obtenons le groupe d'équivalence structurale $\{3,4\}$ et ses conditionnalités, et nous avons constitué le sommet virtuel $(3,4)$. Pour indiquer sa relativité au sommet 1 , nous le relierons par un arc virtuel à 1 . Nous nommerons ce lien virtuel un lien en $\mathrm{Y}$ (il lie 1 et $\{3,4\}$ )

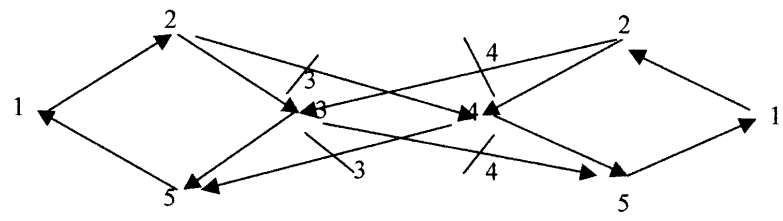

graphe dédoublé

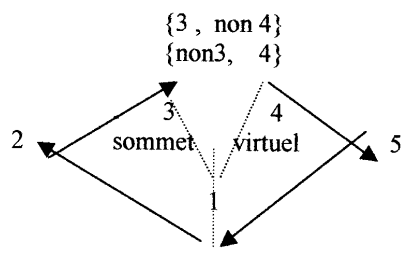

graphe replié et lien en Y 
Notons que le lien en Y revient à re-projeter dans le graphe de relations initial les relations entre les classes que l'on peut tirer de ce graphe initial. En effet nous en tirerons au moins deux classes, celle des sommets qui sont structurellement équivalents, et celle des 3 autres sommets, et nous aurons un lien dans les deux sens entre ces deux classes. Le lien en Y revient à réinscrire l'un de ces liens entre classes en se focalisant sur le sommet 1 , supposé représenter notre acteur de base. Nous pouvons en tirer la conclusion suivante:

Un sommet virtuel apparaît dans un réseau social dès lors qu'on peut établir un lien entre un individu et une classe (d'équivalence structurale), et que le maintien dans le réseau de cet individu dépend précisément de la relation conditionnelle qui assure cette équivalence structurale. L'entité virtuelle «nous», «groupe», «collectif» est donc la re-projection dans le réseau social du rapport des acteurs individuels aux classes issues de leur propre réseau et aux relations conditionnelles qui font jouer le réseau de manière dédoublée.

Mais ne pourrait-on se passer de ce sommet virtuel? Là encore, la simple probabilité du remplacement de 3 par 4 et de 4 par 3 si l'un ou l'autre est défaillant ne suffirait-elle pas? Elle suffirait, s'il s'agit bien d'une double probabilité conditionnelle, donc si la probabilité que 3 soit défaillant active bien 4 et réciproquement. Mais dans ce cas, 1 peut à juste titre tenir 3 et 4 pour structurellement équivalents, et se rapporter à eux comme à une classe de substituabilité, et aucune classe de substituabilité n'est actuelle, puisque seulement un de ses éléments l'est à chaque fois.

Les exemples que nous avons donnés suggèrent des relations de coopération réciproques: si les chasseurs rentrent bredouilles, ils peuvent compter sur l'activité de cueillette des autres pour être nourris. Si les parents doivent travailler aux champs, les enfants sont gardés par leurs frères et sœurs plus âgés, mais si ces frères et sœurs doivent partir pour l'école, un des parents garde les enfants qui restent. Mais la relation de substituabilité conditionnelle n'exige pas une parfaite réciprocité - et d'ailleurs dans le premier exemple, la réciproque n'est pas toujours vraie: si les cueilleurs rentrent bredouilles, ils ne peuvent pas pour autant compter sur l'activité de chasse, plus aléatoire. Les chasseurs dans ce cas ne partent pas forcément à la chasse, mais éventuellement vont tenter des cueillettes plus lointaines. En revanche, la relation de substituabilité conditionnelle n'est pas satisfaite simplement par une relation conditionnelle à sens unique, puisque si la substitution ne pouvait se faire que des cueilleurs aux chasseurs, il suffirait que la cueillette soit un échec pour que l'acteur de base de notre réseau voit sa survie et son maintien dans le réseau mis en danger. Il nous faut donc admettre des relations moins exigeantes qu'une substitution conditionnelle réciproque (les cueilleurs se substituant aux chasseurs en cas d'échec, les chasseurs aux cueilleurs en cas d'échec) mais plus fortes qu'une substitution unilatérale. Les substitutions réciproques peuvent être partielles: les chasseurs en cas d'échec des cueilleurs vont bien se substituer aux cueilleurs, mais pour cueillir, non pour chasser. Il en est de même dans la famille: si les enfants les plus âgés se substituent aux deux parents, il se peut qu'en retour un seul parent se substitue aux enfants les plus âgés. La substitution qui joue le rôle de réciproque peut aussi ne pas être directe, mais médiatisée par des substitutions d'un autre type. Les chasseurs ne vont pas se substituer aux cueilleurs en cas d'échec de la cueillette. La substitution qui peut alors jouer le rôle de réciproque à 
celle des cueilleurs aux chasseurs en cas d'échec de la chasse, c'est une substitution qui a pour condition non plus sur l'échec, mais l'abondance - la relation conditionnelle a changé de condition - et qui exige non pas la substitution des chasseurs aux cueilleurs, mais la transformation du rôle des cueilleurs en celui d'artisans qui savent tirer parti de tous les sous-produits du gibier.

Une question à la fois logique et ontologique se pose alors. Est-il nécessaire pour autant que notre acteur 1 se réfère à une double relation conditionnelle ? Le conditionnel «si...alors » ne se réduit pas à l'implication, et sa sémantique a exigé de recourir à des mondes possibles contrefactuels. Un tel foisonnement ontologique n'est pas interdit - il y a sans doute des interactions sociales qui l'exigentmais pour l'instant nous n'en avons pas besoin. Il suffit de ramener ces conditionnels contrefactuels à ce qu'on pourrait appeler leur base factuelle - des philosophes ont nommés cela leurs « vérifacteurs». Quels sont les états de faits qui valident la proposition «Si 3 ne fait pas l'affaire, alors 4 est mis à contribution, et inversement si 4 ne fait pas l'affaire, alors 3 est mis à contribution»? On pourrait penser à la disjonction exclusive, puisque soit 3 est activé, soit 4 activé, mais pas les deux ensembles. Mais ce serait inexact. Le réseau a bien à sa disposition et 3 et 4 . Nous devons donc revenir à une disjonction inclusive. Cependant il est une forme de la disjonction inclusive qui est insuffisante, c'est celle de la disjonction additive. Elle suppose que, indépendamment de notre choix, 3 est le cas, ou 4. Mais ici c'est du couple 3 et 4 que nous avons besoin. Il nous faut donc un connecteur logique qui porte sur le couple (sur le produit cartésien) et qui cependant traite de manière disjointe 3 et 4 . Un tel connecteur existe, c'est la disjonction multiplicative, nommée «Par» en logique linéaire ${ }^{11}$. L'aspect multiplicatif de ce connecteur exige de croiser des déterminations, au lieu de les tenir pour séparées (on travaille donc sur des produits cartésiens). La disjonction, en sens inverse, introduit un «ou» dont on ne peut pas assurer qu'il va jusqu'au «et». Le comportement de la disjonction multiplicative dans une procédure de preuve est intéressant. Quand on veut tenter de vérifier qu'un énoncé complexe a une preuve, il faut le décomposer en éliminant successivement des connecteurs pour remonter finalement dans l'arbre ascendant de la preuve à des axiomes. Une fois éliminé le connecteur, il s'agit de savoir si les deux sous-formules qu'il reliait peuvent et doivent être elles-mêmes développées à un même niveau de remontée dans la preuve (ce qui est seulement le cas de la conjonction multiplicative ${ }^{12}$ ), ou bien si

11 Dans la logique classique, les deux disjonctions, additives et multiplicatives, sont confondues, parce que l'ajout d'autres règles, l'affaiblissement et la contraction, ne permet plus de repérer la différence entre les deux.

${ }^{12}$ La conjonction multiplicative exige de travailler sur un couple d'éléments, et de les traiter ensemble. La disjonction multiplicative travaille aussi sur un couple d'éléments, mais ne les traite pas ensemble. De manière un peu paradoxale, on l'a nommée un connecteur «synchrone», puisqu'on peut traiter le connecteur quand on veut, cela ne va rien changer. La conjonction additive constitue simplement le couple d'éléments (le produit cartésien), et l'un peut traiter l'un ou l'autre au choix (là aussi, c'est synchrone); la disjonction additive travaille sur un élément, le choix a été fait pour nous et c'est à nous de découvrir quel élément est disponible (ce peut être l'un, ou l'autre, ou les deux, et c'est asynchrone). Cf. J.-M. Andréoli, Logic programming with focusing proofs in linear logic, The Journal of Logic and Computation, 2(3): 297-347, 1992. 
on peut développer une sous-formule, et ne se soucier de développer l'autre que si on en a besoin (c'est la conjonction additive, on a le choix entre l'un ou l'autre développement), ou bien enfin si l'on peut développer l'une séparément à un niveau, mais à condition toujours d'avoir à développer l'autre à un autre niveau de la remontée dans la preuve. La disjonction multiplicative correspond à ce dernier comportement. Elle se comporte donc comme si le développement d'une sousformule était conditionné au développement ultérieur de l'autre sous-formule, et réciproquement, et ce, alors que le connecteur Par est bien moins complexe ontologiquement qu'un conditionnel contrefactuel avec sa réciproque. Le connecteur Par mime donc sans recourir au conditionnel contrefactuel un comportement de conditionnalité qui donne lieu à réciproque ou plutôt alternance à un moment où un autre. Nous pourrions donc nous satisfaire de ce connecteur, au lieu de devoir recourir au conditionnel contrefactuel, car les ontologies proposées pour correspondre au conditionnel sont très (trop?) riches, puisqu'elles impliquent des emboîtements de mondes possibles contrefactuels.

Quel est alors l'avantage de cette conception des collectifs?

Le premier avantage est de définir les conditions d'émergence de cette entité collective. C'est simplement un réseau de relations conditionnelles: une fois un circuit établi dans un réseau, le maintien dans le circuit de chaque individu de base dépend de l'intégration dans le réseau d'au moins une classe d'équivalence conditionnelle.

Le second avantage est de nous libérer de l'image d'un nous comme entité surplombante au dessus des individus. Dans le cas hyper-distribué que nous avons évoqué (lui aussi un cas fictif, cependant), chaque acteur de base est dépendant, pour son maintien dans le réseau, de l'équivalence conditionnelle propre à une classe d'autres acteurs, mais chacun étant dépendant d'une équivalence conditionnelle différente, il n'existe pas d'entité unique de laquelle chacun dépende. Les liens en Y ne convergent pas vers une sorte de centre, ils ne transforment pas le réseau en une structure en étoile autour du sommet virtuel. Il suffit que toutes les classes d'équivalence conditionnelles nécessaires à un ou plusieurs acteurs possèdent de proche en proche des éléments communs, et que les acteurs de base soient en réseau, qu'existent dans ce réseau des circuits entre acteur de base et groupes d'équivalence conditionnelles, pour que nous ayons bien affaire à une entité virtuelle collective. Simplement cette entité collective reste répartie sur les différentes classes d'équivalences conditionnelles. Son unité reste distribuée.

On évite d'ailleurs aussi la structure en étoile dans un autre cas limite, celui où chaque acteur est dépendant de la même classe d'équivalence conditionnelle que les autres, à trois acteur près. Ici il y a bien une sorte de noyau du collectif, mais il peut être tellement étendu et très probablement tellement riche en dépendances diverses qu'il peut difficilement être tenu pour une «unité » centrale. De toutes manières, les relations conditionnelles assurées pour chaque individu de base restent toujours différentes, au moins sur quelques points, et la perspective de chaque individu, qui est aussi une relation de dépendance conditionnelle, reste différente de celle d'un autre. A fortiori l'entité collective reste distribuée quand on se situe en dehors de ces cas limites. C'est seulement dans le premier cas limite, celui où n'existerait que des substituabilités conditionnelles différentes pour chaque acteur, et où ces acteurs ne participeraient pas à d'autres substituabilités, 
que nous hésiterions à parler d'entité collective, puisque cela n'aurait de sens à chaque fois que dans des perspectives individuelles séparées.

Mais précisément, dira-t-on, l'existence d'une entité sociale et collective n'exige-t-elle pas que chacun de ses membres se réfère à «la même » entité collective? L'avantage de notre présentation est de montrer que ce n'est nullement nécessaire, et d'indiquer comment on peut échapper à cette contrainte. Chacun se réfère à ce que nous appelons une classe d'équivalence conditionnelle, mais il ne suppose pas que les autres se réfèrent à «la même». Il suppose au contraire qu'il existe des individus qui ont un rapport direct avec les activités de cette classe d'équivalence (ce sont les individus qui agissent en interaction directe avec les coordinations réalisées dans cette classe), et donc pour qui il n'y a pas «équivalence». Il suppose seulement que les classes d'équivalence conditionnelle auxquelles les autres se réfèrent font partie d'un même réseau, et souvent d'un même circuit. Si la notion de circuit permet une clôture, celle de réseau reste ouverte: les frontières d'un réseau sont difficiles à définir. Et dans une société complexe, avec une division sociale du travail élaborée, avec des institutions, notre acteur de base suppose que ces classes d'équivalence de base sont ellesmêmes des éléments de classes d'équivalence conditionnelle d'ordre supérieur. Chaque individu se rapporte donc au collectif comme à une entité dont l'unité tient à sa connexité et non pas à son unification en un seul foyer. C'est d'ailleurs ce que montrait le fait que ce qui tenait lieu dans notre graphe de l'entité collective, c'était un lien en Y. Dans le graphe d'un groupe réel, il y aurait plusieurs liens en Y, et le groupe existe en tant qu'entité sans que les nœuds de ces liens en Y doivent pour cela fusionner.

\section{COLLECTIFS VIRTUELS ET REPRÉSENTATIONS}

Il nous faut maintenant revenir sur la relation de cette entité collective virtuelle avec les représentations individuelles.

La position que nous avons prise concernant les conditionnels contrefactuels suggère que nous soyons prudents quant au rôle des représentations, qui elles aussi exigent une ontologie foisonnante. De fait, les conditions d'existence d'une entité collective virtuelle, telles que nous les avons extraites, n'impliquent pas la nécessité d'une référence à l'idée de représentation. Si le circuit d'activités fonctionne, et qu'il comporte en son sein une classe d'équivalence ou de substituabilité conditionnelle, alors en ce qui concerne ce fonctionnement particulier, à savoir le maintien de l'individu de référence dans le circuit, il y a bien entité virtuelle. On pourrait parler d'existence fonctionnelle de cette entité. Un observateur extérieur pourrait déduire cette entité virtuelle alors même que l'individu en question ne se la représenterait pas. L'existence d'une représentation sociale dépend de l'existence de l'entité collective virtuelle et non l'inverse.

En fait, les représentations sociales sont elles-mêmes des classes d'équivalences ou de substitutions conditionnelles. Une représentation (au sens social du terme, mais à vrai dire on voit mal quel autre sens pourrait être proposé, sauf à recourir à l'idée de représentations purement privées, et qu'il serait alors difficile de simplement ré-évoquer dans une situation différente) est quelque chose qui 
permet de contrôler une activité ou un ensemble d'activités et qui peut être transféré d'un individu à l'autre tout en continuant à se référer à une même activité ou un même ensemble d'activités, mais cette fois vue sous la perspective de cet individu au lieu de celle du premier (et donc, contrôlable de la situation du second au lieu de celle du premier) ${ }^{13}$. On retrouve ici la substituabilité des individus pour une activité donnée, et cette substituabilité est bien encore ici conditionnelle, non plus cette fois à la défaillance d'un acteur, qu'un autre doit remplacer, mais au remplacement impliqué par le transfert de perspectives. Rien n'assure que l'expérience privée des représentations soit la même chez les deux individus, tout ce qui importe c'est que malgré le transfert de perspectives la même activité soit assurée (ou le même ensemble d'activités). Dans nos activités, dès que nous utilisons des représentations sociales, et que nous sommes membres d'un circuit d'activités, nous présupposons donc l'existence d'une entité collective.

La question qui se pose est maintenant de savoir ce que peut apporter aux activités sociales la représentation de cette entité collective - et non plus comme précédemment la présupposition de l'entité collective par des représentations sociales qui ne portent pas sur elle. Nous disposons déjà des éléments de la réponse, puisque nous avons envisagé les diverses formes d'une entité collective distribuée. Nous avons aussi rappelé que la frontière d'un réseau est difficile à définir. Supposons qu'il soit utile au maintien des individus dans les circuits de leur réseau social de pouvoir distinguer ce réseau d'autres réseaux sociaux, ne serait-ce que pour mieux organiser leurs interactions. Concevoir la représentation sociale de ce réseau comme une entité collective correspondant à une classe d'équivalence conditionnelle nous permet de surmonter le problème tenant à cette difficulté de définir la frontière d'un réseau. Ce problème tient à ce que pour un individu la frontière est déterminée d'une certaine manière, pour un autre d'une autre, puisque chacun a des réseaux d'interaction différents. Il en est de même, nous l'avons vu, pour les classes d'équivalences conditionnelles différentes qui sont pourtant le fondement de l'entité collective du groupe social.

Une représentation de cette entité collective est alors ce qui permet la substituabilité d'un réseau à un autre, d'un noyau de classes d'équivalence à un autre, la substitution étant conditionnelle au déplacement d'une perspective individuelle à l'autre, à condition que ces individus soient reliés par des circuits qui font euxmêmes partie de réseaux. Et les réseaux dont chacun doit faire partie sont précisément ceux qui sont reliés par ces substituabilités conditionnelles. L'existence de représentations individuelles du collectif est alors une condition du maintien des réseaux individuels dans l'orbe des substitutions qui correspondent à l'entité collective. Cela permet que ces différents réseaux individuels se relient tous à une même entité collective, qui peut donc se différencier d'autres entités collectives, au lieu que chaque réseau particulier tende à s'autonomiser. Etant donné le concept de représentation sociale tel que nous l'avons défini plus haut (une représentation sociale assure une substituabilité conditionnelle avec changement de perspective individuelle et présuppose une entité collective), nous voyons que les

${ }^{13}$ On pourrait ici rendre compatibles Sperber et Lahire: les représentations transmissibles du premier ont bien des dispositions sociales au sens du second. 
représentations individuelles du collectif, en tant que représentations sociales, vont forcément elles-mêmes relever d'une représentation collective possible: celle qui assure la substituabilité des représentations individuelles, qui présuppose l'entité collective ainsi représentée, et qui limite la dispersion de cette entité.

Le processus de constitution d'une représentation d'une entité collective que nous venons de définir peut aisément se transposer à un niveau supérieur. Cela selon différentes modalités. Ainsi la représentation d'une entité collective peut englober diverses entités collectives, qui ne sont elles-mêmes que présupposées par des activités en réseau sans donner lieu à représentations. Ou inversement, diverses représentations d'entités collectives peuvent donner lieu fonctionnellement, sans représentation d'ordre plus élevée, à une entité collective d'ordre plus élevé qu'elles présupposent. Ou encore, cette entité collective issue de plusieurs représentations collectives peut elle-même donner lieu à représentation collective.

Les représentations sont ici ramenées à des virtualités, c'est-à-dire au type d'entité engendrée par des activités qui sont substituables conditionnellement les unes aux autres. Une société peut se mettre en place si ces activités fonctionnent en circuit de manière suffisamment régulière et si ces substituabilités conditionnelles fonctionnent elles aussi de manière suffisamment régulière. L'origine d'une société, ce sont donc à la base la capacité de certaines activités à s'engrener les unes dans les autres, la capacité du circuit de ces activités à s'entretenir, et la capacité de certaines activités à se substituer les unes aux autres de manière conditionnelle. Les représentations n'en sont la condition nécessaire qu'à partir d'un certain degré de complexité des circuits, en particulier quand il faut différencier le réseau lié à ces circuits d'autres réseaux. Mais là encore, elles fonctionnent de la même manière que les substituabilités conditionnelles gagées sur des activités actuelles. Cela nous amène à une vision des sociétés à la fois fonctionnelle (au simple sens de: «ça se trouve fonctionner selon tel circuit ou telle substitution d'activités ») et dispositionnelle (puisqu'une substituabilité conditionnelle implique une disposition à déclencher une activité conditionnellement à l'absence d'une autre). Mais ces dispositions peuvent être décrites dans les termes des activités actuelles qui se substituent l'une à l'autre (en utilisant en particulier le connecteur «Par», la disjonction multiplicative).

\section{REPRISE DE QUELQUES THÉORIES SOCIALES}

Nous pouvons alors reprendre différentes hypothèses concernant l'existence des collectifs, à chaque fois en les révisant: l'hypothèse représentationnelle par «common knowledge » (Lewis, Gilbert, Pettit) l'hypothèse de rationalité individuelle en contexte (Boudon), l'hypothèse dispositionnelle et contextuelle d'habitus et de capital culturel (Bourdieu), la notion de cité ou de régime social proposée par Boltanski et Thévenot, et finalement l'analyse de la galère dans les banlieues proposée par Dubet ${ }^{14}$.

${ }^{14}$ Nous ne suivons donc pas l'ordre chronologique. Ainsi les analyses de La galère datent des années 80 , et nous ne les prenons pas comme une théorie sociale nouvelle concernant les collectifs, mais comme un exemple qui démontre que les acteurs sociaux ne se pensent pas autorisés à avoir des 
Notre conception des représentations collectives nous dispense de l'hypothèse de «common knowledge » - où chacun doit se représenter que les autres se représentent que tous se représentent, et cela à l'infini. Les représentations collectives impliquent seulement que les changements de perspectives d'un individu à l'autre maintiennent les classes d'équivalences conditionnelles entre activités. Cela se vérifie dans la pratique, au niveau du circuit des activités, et non pas dans un examen de la présence des représentations de premier, de second, de nième ordre, examen d'ailleurs impossible.

L'hypothèse de rationalité individuelle en contexte doit être en quelque sorte démultipliée, selon différents types de rationalité. Un premier type de rationalité se réduit à la fiabilité du circuit d'activités, qui continue à s'entretenir et survit dans l'évolution. Ici le contexte, c'est l'environnement écologique de ce circuit d'activités. Les individus sont seulement les porteurs des activités. Un individu se constitue comme être social quand il peut se poser comme le foyer de plusieurs liens en Y, de plusieurs relations à des équivalences conditionnelles entre activités. Cela exige une certaine capacité de représentation, celle qui consiste pour l'acteur, en fonction de sa propre perspective, à reconnaître la convergence de ces équivalences conditionnelles. L'individu est alors au foyer de différents contextes, puisque les équivalences conditionnelles peuvent appartenir à différents circuits et réseaux qui constituent autant de contextes. Cela n'exige pas de représentation d'entités collectives ni même de représentations sociales pour autant (là encore, nous ne connaissons pas de société réelle aussi pauvre en représentations).

En revanche, pour en arriver au stade des «bonnes raisons », il faut disposer de représentations sociales, puisque ces bonnes raisons sont communicables et qu'elles permettent à des individus différents de contrôler des activités similaires. Les bonnes raisons sont cependant contextuelles: elles ne valent que dans certains circuits et réseaux, ou dans une classe d'équivalence conditionnelle entre réseaux. Jusqu'à ce stade nous n'avons donc pas besoin de représentations collectives. Mais il est assurément des activités sociales pour lesquelles elles sont nécessaires, ne serait-ce que pour les activités politiques, par exemple pour rendre rationnelle l'activité d'aller voter.

Nous utilisons la notion de disposition et nous la retrouvons chez Bourdieu ${ }^{15}$. Admettons qu'elle puisse aussi chez lui se ramener à celle de relation conditionnelle. Les notions dont nous pourrions donner une version révisée ne seraient alors pas celle de disposition, mais celles d'habitus, et de capital symbolique ou culturel. Commençons par la notion d'habitus. Un circuit d'activités ne se met en place que s’il se répète régulièrement. Cela suffit pour que chaque individu ait certaines attentes, mais cela n'épuise pas la notion d'habitus. Elle exige que les individus acquièrent peu à peu une panoplie d'activités substituables condition-

représentations collectives unifiées si leur entité collective ne l'est pas. Par ailleurs il nous semble possible d'utiliser les concepts proposés pour relier Parsons, et en particulier sa notion de rôle, mais il y faudrait de plus amples développements.

15 Par exemple chez Bourdieu, Le sens pratique, Les éditions de minuit, 1980, et son commentaire par Bernard Lahire, L'esprit sociologique, Editions la Découverte, 2005. 
nellement aux activités des autres et à un contexte pragmatique donné. Il faut donc que notre acteur de base puisse se retrouver dans la position de l'acteur qui doit se substituer à un autre ou qui ait besoin qu'on se substitue à lui pour telle activité, et qu'il puisse se retrouver impliqué dans plusieurs situations qui exigent aussi des substituabilités conditionnelles. C'est seulement à cette condition qu'il va pouvoir développer un habitus. Un habitus n'est donc pas seulement l'apprentissage d'une activité, c'est aussi l'apprentissage de complémentarités et de coordinations, c'est l'apprentissage d'une variété de telles coordinations, ainsi que celui des manières de choisir la coordination appropriée à la situation - ce qui implique par là même l'apprentissage d'une identification des contextes qui définissent la pertinence de telle coordination (coordination qui peut être solidaire, mais aussi compétitive ou conflictuelle). Nous sommes ici parti de la perspective d'un acteur. Mais nous avons vu qu'une perspective ainsi reliée à une équivalence conditionnelle impliquait une entité collective. D'après notre interprétation de l'habitus et les définitions données plus haut, on vérifiera que tout acquisition d'habitus suppose la constitution d'entités collectives variées et même d'une entité collective de second ordre qui les subsume (puisqu'il faut pouvoir relier diverses substituabilités conditionnelles).

Les notions de capital symbolique et de capital culturel sont plus controversées, et plus difficilement définies. Leur pouvoir explicatif semble être pour une part de rendre compte de ce que pour un groupe, un ensemble d'activités et de coordinations est jugé possible, alors qu'un autre ensemble ne l'est pas, et que pour un autre groupe, cette séparation porte sur des ensembles d'activités différents. D'autre part, il s'agit aussi de comprendre comment les valorisations des activités s'organisent différemment selon les groupes, et, dans un cercle d'acteurs et d'activités donné, quelles valorisations d'activités donnent à leurs acteurs une capacité (une chance supérieure, une disposition) à obtenir plus aisément des autres certaines coordinations.

Pour pouvoir juger possibles certaines activités et pas d'autres, les individus ont assurément besoin de représentations sociales. Or ces représentations portent alors non pas seulement sur des substituabilités conditionnelles, mais surtout sur des non substituabilités. L'introduction de ces «non substituabilités» permet de présupposer l'entité collective commune, sans exiger une représentation d'une entité collective commune aux différents groupes, ou du moins sans avoir à donner à la représentation collective commune un rôle autre que secondaire. Cela permet alors de ne pas avoir à définir positivement et dans l'absolu les valeurs propres au groupe, et de les utiliser seulement de manière différentielle, en portant attention à ce qui n'est pas transférable et substituable, quand il s'agit de se comprendre comme faisant partie d'un groupe et non d'un autre.

Mais ces différentiels ne sont pas seulement définis de manière externe, ils peuvent aussi l'être de manière interne au groupe. Un acteur enrichit son capital social si il peut assurer des coordinations - rendre efficaces des substituabilités conditionnelles- qui avaient peu de chances sans lui de s'établir. En un sens, il élargit l'ensemble des substituabilités conditionnelles, en un autre, il établit à son profit une non substituabilité par rapport aux autres, puisque c'est lui, et non pas les autres, qui a permis cette coordination inespérée. Il se pourrait que le capital symbolique s'acquière par cette sorte d'échange d'une substituabilité conditionnelle supplémentaire pour les autres acteurs contre un différentiel de substituabilité en faveur du coordinateur. Une fois ce différentiel reconnu, il peut être réuti- 
lisé pour favoriser d'autres coordinations soit qui sont directement à l'avantage du coordinateur, soit qui augmentent ce différentiel, et ainsi de suite.

Cette reconstruction de la notion de capital symbolique nous permettrait de comprendre pourquoi il n'est pas nécessaire que les acteurs se représentent ces processus de gain de substituabilité et de différentiel comme tels (il suffit que les entités collectives impliquées soient ainsi constituées), et pourquoi malgré cela le contenu signifiant de ces processus ait bien le statut de ce que l'on trouve souvent désigné par le substantif «le symbolique». A notre sens, «le symbolique» désigne non pas une entité collective, mais une marque place pour cette relation intime entre une entité collective et les représentations sociales qu'elle permet. Dans le cas d'une représentation d'entité collective proprement dite, il y a identité entre l'entité, le symbolique et le contenu de la représentation, mais la plupart du temps, il s'agit simplement d'un lien (comme l'indique le terme même de «symbole») entre entité collective, ensemble de substituabilités, et représentations, et non pas d'une convergence sur un même référent.

Notre perspective permet bien un «retour aux acteurs », puisqu'elle exige à chaque fois de partir de la fonction qu'a une coordination pour le maintien dans le réseau d'un des acteurs du réseau. Mais elle fait passer leurs activités avant leurs représentations. Elle permet aussi de soutenir que les acteurs peuvent dépendre de coordinations qu'ils ne contrôlent nullement, et que ces dépendances et les entités virtuelles qu'elle implique s'imposent à eux dès lors qu'ils utilisent le résultat de ces coordinations. Comment pouvons nous dans ce cadre reconstituer la perspective de Boltanski et Thévenot, sensibles aux valeurs que les acteurs sociaux expriment, et soucieux d'en montrer la cohérence dans ce qu'ils appellent des cités ou des régimes d'action ${ }^{16}$ ? Il faut noter qu'en explicitant la cohérence de chacune de ces cités, Boltanski et Thévenot vont plus loin que les acteurs eux-mêmes. Les acteurs se satisfont de présupposer une entité collective et de construire des représentations différentielles entre régimes d'action. Expliciter l'entité collective en tant que «cité» et concevoir les propriétés différentielles ainsi représentées comme étant les attributs de cette entité est peut-être aller plus loin que les acteurs. Cela a cependant un avantage quand on veut mettre mieux en évidence les conflits entre régimes d'action, par exemple les disputes concernant la durée de vie souhaitable d'une machine (une machine à laver, par exemple), disputes nées du conflit entre le souci de la qualité de la production du point de vue technique et le souci de renouvellement des biens du point de vue marchand. Mais ces disputes peuvent naître et prendre sens dès que les acteurs s'aperçoivent qu'un bien peut ressortir de différents circuits, de différentes coordinations, et que les conditions de clôture ou de maintenance des différents circuits peuvent être contradictoires. Pour cela, il faut qu'ils puissent eux-mêmes adopter les points de vue des différents circuits et donc qu'ils participent à plusieurs circuits et à plusieurs entités collectives virtuelles. Il est possible que ce que Walzer avait appelé «sphères de justice» corresponde non pas à des entités collectives, mais aux exigences de réciprocité conditionnelle qui fondent ces entités collectives.

\footnotetext{
${ }^{16}$ Luc Boltanski et Laurent Thévenot, De la justification, Paris, Gallimard, 1991.
} 
Enfin nous pouvons trouver une confirmation de notre principe de la priorité de l'entité collective sur les représentations collectives dans le travail de Dubet sur les jeunes des banlieues, sur cette situation qu'il nomme avec eux «la galère ${ }^{17}$, confirmation ${ }^{18}$ d'autant plus claire que Dubet ne partait pas avec cette hypothèse. Il était parti d'un programme inspiré par la problématique de Touraine, qui consistait à rechercher les acteurs collectifs émergents, les promoteurs de nouveaux mouvements sociaux. Pour qu'émerge un tel acteur, Dubet rappelle qu'il faut des «points d'appui », des constitutions de solidarités, d'associations de lutte, la participation d'intellectuels. C'est dire que doit se constituer un réseau plus riche de coordinations, de coopérations, que davantage de substituabilités conditionnelles doivent pouvoir faire réseau. La métaphore du mouvement social comme «acteur» impliquerait que ces coordinations se coordonnent les unes aux autres. Cela peut s'interpréter comme la convergence des liens en Y sur une même entité collective, ce qui correspond bien à la métaphore de l'acteur collectif. Mais il est plus plausible d'y voir des substituabilités conditionnelles de second ordre, qui jouent donc elles-mêmes entre différents types de coordinations et coopérations.

La découverte de Dubet, c'est que les jeunes des quartiers ne veulent justement pas d'une telle structure, qui introduit forcément une hiérarchie entre niveaux, entre coordinations de premier ordre et coordinations de second ordre. Ils ne veulent pas que leurs activités soit «récupérées » par des acteurs qui se poseraient en représentants des entités collectives que présupposent les coordinations éparses entre jeunes, et qui voudraient rassembler ces entités et leurs coordinations dans une structure plus centralisée. Ce qui va de pair avec ce refus d'une structure en niveaux, c'est aussi le refus de se donner un adversaire social spécifique (comme une bourgeoisie obstacle au prolétariat), voir même de poser le groupe des jeunes comme en conflit de génération avec le groupe des plus vieux. Les jeunes de la galère ont cependant d'eux-mêmes une représentation collective, qui correspond à une structure éloignée du cas limite que serait la dépendance de chacun par rapport à un noyau central, et plus proche de l'autre cas limite, celui d'une nébuleuse où chaque individu se rapporte à des équivalences ou substituabilités conditionnelles différentes, mais qui sont toutes en réseau. L'unité collective des jeunes des banlieues reste très distribuée. La découverte qui reste quelque peu implicite dans le travail de Dubet est que c'est précisément par la différence entre cette structure très distribuée et d'autres structures plus classiques (les ouvriers, les cadres, la police, les politiques, les entreprises, l'institution scolaire, etc.) que les jeunes peuvent se représenter leur identité collective.

Nous l'avions dit, une des raisons pour qu'une représentation collective apparaisse est qu'il soit utile, pour le maintien des individus dans leur réseau, qu'ils puissent le distinguer d'autres réseaux sociaux. Mais pour qui est-ce utile? Ici, c'est sans doute plus utile, différentiellement, pour les autres réseaux sociaux que pour les jeunes des banlieues - ils sont vite repérés par la police, ils n'arrivent pas à se faire embaucher, et ce au profit de membres d'autres groupes sociaux. De même que la notion de capital symbolique nous obligeait à introduire la notion de «non-substituabilité », l'analyse de la galère nous oblige à introduire la notion

${ }^{17}$ François Dubet, La galère: Jeunes en survie, Points Fayard, 1987

${ }_{18}$ A condition bien entendu d'accepter notre interprétation. 
d'une utilité de se différencier d'autres réseaux qui reste négative comme utilité interne au groupe considéré, et qui n'est positive que pour les autres groupes dont il se différencie. Nous pouvons alors parler pour ce groupe de désutilité différentielle. D'autres groupes ont par le passé constitué leur unité collective au moins partiellement comme une fonction de leur désutilité différentielle (les ouvriers, ou même les cadres industriels et de marketing par rapport aux décideurs financiers), mais ils ont aussi, du moins pour les ouvriers, construit un réseau plus dense entre les solidarités et appuis locaux.

Le souci des jeunes des banlieues de ne pas se laisser entraîner dans ce scénario éprouvé peut d'ailleurs tenir aussi à ce que ce scénario leur est suggéré de l'extérieur, venant de délégués de la société même qui fait de leur différence une désutilité. On leur suggère d'être autonomes, de s'organiser en associations et de se donner des objectifs de lutte, mais ces suggestions, quelles que soient les bonnes volontés et les bonnes intentions de ceux qui les font, viennent d'une société qui ne les intègre pas dans ses coordinations usuelles (travailler, gagner de l'argent, consommer). Si la représentation par les jeunes de leur propre entité collective reste aussi distribuée, c'est parce qu'une autre représentation ne viendrait pas vraiment d'eux, et dépendrait donc de facteurs externes à leurs réseaux de coopérations, à leurs réseaux de substituabilités conditionnelles. Ils refusent donc une représentation collective qui ne correspondrait pas à la structure propre de leur entité collective. Nous trouvons aussi dans «la galère» une nouvelle vérification du principe énoncé plus haut: c'est l'entité collective qui commande la représentation collective et non l'inverse.

Nous avons tenté une sorte de déconstruction des représentations prototypiques d'un collectif, comme centre d'un réseau ou clôture d'un ensemble. Nous avons montré que le statut des entités collectives relevait du virtuel, et que pour donner une existence à ce virtuel, il fallait qu'il manifeste des dépendances conditionnelles entre activités actuelles. Les liens sociaux reposent sur de telles dépendances conditionnelles. L'entité collective qu'elles présupposent peut avoir une structure beaucoup plus distribuée que celle de nos représentations prototypiques du collectif. C'est cette structure que le sociologue peut penser mettre partiellement au jour, en examinant les réseaux d'interactions aussi bien que les représentations sociales. 